\title{
Manifestation of the geometric phase in neutron spin-echo experiments
}

\author{
W. H. Kraan, ${ }^{1}$ S. V. Grigoriev, ${ }^{1,2}$ and M. T. Rekveldt ${ }^{1}$ \\ ${ }^{1}$ Department R3, Faculty of Applied Sciences, Delft University of Technology, $2629 \mathrm{JB}$ Delft, The Netherlands \\ ${ }^{2}$ Petersburg Nuclear Physics Institute, Gatchina, St. Petersburg 188300, Russia
}

(Received 8 March 2010; published 14 July 2010)

\begin{abstract}
We show how the geometric (Berry's) phase becomes manifest on adiabatic rotation of the polarization vector in the magnetic field configuration in the arms in a neutron spin echo (NSE) experiment. When the neutron beam used is monochromatic, a geometric phase collected in one spin-echo arm can be exactly compensated in the other arm either by an opposite geometrical rotation or by adding/subtracting a dynamic (Larmor) phase. This is not possible in a white beam, because, contrary to the dynamic phase, the geometric phase is independent of wavelength. Therefore, the NSE pattern can be disturbed. We demonstrate that adiabatic resonant spin flippers inherently produce a geometric phase which can influence the performance of NSE setups based on such flippers. This effect can be avoided by a proper mutual symmetry of the gradient fields in these flippers.
\end{abstract}

DOI: 10.1103/PhysRevA.82.013619

PACS number(s): 03.75.Dg

\section{INTRODUCTION}

In neutron spin-echo (NSE) experiments the precession phase of the polarization vector accumulated while the beam runs through some field configuration is compensated by the precession in a following identical configuration, with reversed precession. The degree of the resulting polarization is commonly measured by off-setting the NSE condition by inserting a small so-called phase coil in one NSE arm and varying its field. One measures the amplitude of the damped oscillating signal (for the whole spectrum) thus obtained: the so-called spin-echo group. Presently, several instruments in the world for this purpose are operational [1,2]. Here we discuss the NSE experiment in a setup installed in a pulsed white beam, where each NSE arm consists of two adiabatic radiofrequency (RF)/gradient flippers as described for the first time by Bazhenov et al. [3].

As is well known (see, e.g., Ref. [4]), each component of the spinor $|\chi\rangle$ describing a neutron flying through the magnetic field configuration $\vec{B}(t)$ (with $t=x / v$ ) of an NSE arm, picks up a phase $\mathrm{e}^{i m \phi}$ where $\phi=\int_{0}^{L}|B|(x) d x(m= \pm 1)$, the socalled dynamic phase. Since the time spent in the magnetic field is proportional to the wavelength $\lambda$, this phase is proportional to $\lambda$.

When a field configuration is such that the resulting vector $\vec{B}$ (as seen by the flying neutron) describes a closed loop, each spin state also collects a geometric phase $\phi_{g}$, which is independent of wavelength. Long ago Berry [5] stated that $\phi_{g}$ is equal to the solid angle subtended by this closed loop. This has been verified for a polarized thermal neutron beam flying through a twisted field $[4,6]$ and through a rotating field [7] and also with cold neutrons [8]. It has been argued that the noncommuting properties of two identical successive neutron spin flippers with different orientations will lead to an observable geometric phase shift effect [9], which has been experimentally verified [10]. The geometric phase was discussed also in contexts outside neutron beams [11], e.g., propagation of light in a fiber [12].

Many studies deal with single-state experiments in an interferometer with different magnetic field configurations along the two paths, in some studies giving different geometric phases $[10,13,14]$. A direct example is the separation geometric/dynamic phase effected by a relative rotation/translation of two $\pi$ flippers installed in the two pathways [15]. An example with analogy to our work with a twisted coil is an interference experiment with fields in the two paths with (adjustable) opposite twist in search for the $4 \pi$ symmetry of the spinor [16]. A situation comparable with interferometry arises when adiabatic/RF flippers are set at flip probability $<1$ : then a coexistence between flipped and nonflipped neutrons states and their interference appears in a single beam line [17].

Recently, the geometric phase received renewed attention in the field of quantum information processing due to its presumed resilience against noise perturbations. In this context its stability for trapped ultracold neutrons subjected to field fluctuations was demonstrated [18].

In our NSE setup the dynamic phase is built up in the "zerofield precession" mode over the path between two adiabatic $\mathrm{RF} /$ gradient flippers, i.e., at twice the rate corresponding to the DC field in the flippers [19]. When such a flipper is viewed in a rotating coordinate system in a way as wass done long ago in NMR theory [20], the field resulting from its (DC) gradient and RF coil can be modeled with a field of strength $A$ rotating uniformly by $\pi$ over its length. One could imagine a NSE arm with two such flippers in which the field rotates over full $2 \pi$, hence a neutron flying through it will pick up a geometric phase.

In an NSE experiment, where the net phase is $\approx 0$, the phase $\phi_{g}$ becomes relevant. Our NSE groups, measured with a "white" neutron beam, showed a slight asymmetry. Differentiated to $\lambda$ the NSE condition was found not to coincide for all $\lambda$, presumably due to a geometric phase picked up by the polarization vector $\vec{P}(t)$ in one or both NSE arms. It is the aim of this article to find and analyze the geometric phase $\phi_{g}$ in various modifications of our NSE setup. These modifications (in one NSE arm only, keeping the other arm unaltered) include (i) varying the phase difference between the RF fields in the flippers, (ii) reversing the gradient field in one and both flippers, and (iii) varying the amplitude $A$ of the rotating field in one flipper. For reference we describe a trivial way to produce a geometric phase: transmitting the beam through a DC coil, twisted over $2 \pi$.

The layout of this article is as follows: in Sec. II we show how the NSE pattern for a setup as described above is disturbed 
when a net geometric phase remains. In Sec. III we calculate the precession phase through one adiabatic/RF flipper and through a full NSE arm consisting of two such flippers. We discuss how this phase behaves as a function of the adiabaticity parameter $k_{a}$ (the ratio between the precession frequency $\gamma A$ in a field of strength $A$ and the change rate of the field direction, as seen by the flying neutron) and how it changes as a result of the modifications just mentioned. In the end we discuss the precession phase after a $2 \pi$-twisted DC coil. After a description of our experimental setup in Sec. IV, we give NSE patterns obtained, applying the modifications mentioned above. This section is completed with NSE patterns obtained with the twisted DC coil. Finally, there is a conclusion.

\section{DISTURBANCE OF NSE BY GEOMETRIC PHASE}

Let the precession phase $\phi_{1}$ in the first arm of a NSE setup be composed of a $\lambda$-dependent dynamic term and a $\lambda$-independent geometric term: $\phi_{1}=\Phi_{1} \lambda+\phi_{g 1}$. In general, the precession $\phi_{2}=\Phi_{2} \lambda+\phi_{g 2}$ in the second arm will not cancel $\phi_{1}$. To find NSE, a phase coil is inserted in this arm, making a field over some length along the beam and thus a dynamic phase proportional to this field.

Let the dynamic phase cancel for the field value $B_{f}$ in this coil, so $\Phi_{1} \lambda=\left(\Phi_{2}-c_{P h} B_{f}\right) \lambda$ for all $\lambda$, hence $\Phi_{1}-\Phi_{2}+$ $c_{P h} B_{f}=0$. ( $c_{P h}$ is a constant characterizing the coil, not to be further specified.) When the field is varied $\Delta B$ away from $B_{f}$, a net phase $\varphi_{\text {net }}(\lambda)$ remains, equal to $\left[\Phi_{1}-\Phi_{2}-c_{P h}\left(B_{f}+\right.\right.$ $\lambda \Delta B)]+\phi_{g 1}-\phi_{g 2}=\lambda \Delta B+\phi_{g 1}-\phi_{g 2}$.

Defining the net geometric phase $\phi_{g}=\phi_{g 1}-\phi_{g 2}$, the measured polarization $P \equiv \cos \varphi_{\text {net }}(\lambda)$ is:

$$
P(\Delta B, \lambda)=\cos \left(\phi_{g}-c_{P h} \lambda \Delta B\right) .
$$

The top row of Fig. 1 is a map of $P(\Delta B, \lambda)$ with net geometric phase $\phi_{g}$ equal to $0, \pi / 2, \pi$ and (arbitrary) 0.77 . In the bottom row $1 / \lambda$ is plotted instead of $\lambda$ : then the lines separating regions with $P>0$ and $<0$ become straight. It is seen that an (inadvertent) net overall geometric phase can turn the map from symmetric into antisymmetric in $\Delta B$. For an
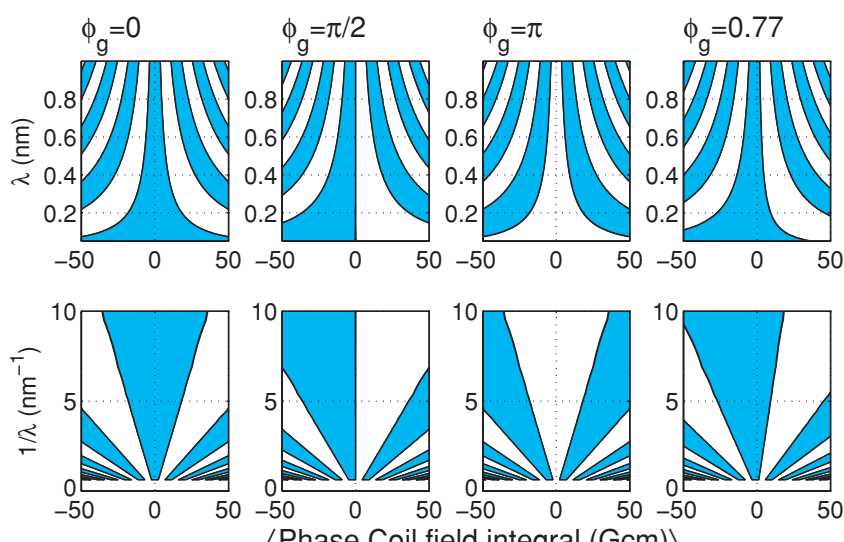

FIG. 1. (Color online) NSE scan in a setup with net geometric phase $\phi_{g}=0, \pi / 2, \pi$ and (arbitrary) 0.77 : maps of the polarization $P(\Delta B, \lambda)$ as a function of phase coil field and wavelength [Eq. (1)] (top row) and transformed to $P(\Delta B, 1 / \lambda)$ (bottom row). (Dark, $P>$ 0 ; white, $P<0$.) arbitrary geometric phase there is no unique phase coil field where $P$ has the same phase for all $\lambda$.

\section{PRECESSION PHASE AFTER ONE NSE ARM}

To understand the NSE experiments described in Sec. IV, we will first calculate the (dynamic and geometric) precession phase $\phi_{f f}$ acquired by the polarization vector $\vec{P}$ while the beam runs through one NSE arm, composed of two adiabatic RF flippers, located a distance $L$ apart.

The behavior of the neutron spin in a beam running through a field $\vec{B}(\vec{r}, t)$ is most generally described by the timedependent Schrödinger equation $i \hbar(\partial / \partial t) \psi(\vec{r}, t)=\mathcal{H} \psi(\vec{r}, t)$, where

$$
\mathcal{H}=\frac{p^{2}}{2 m}-\frac{1}{2} \hbar \gamma \vec{\sigma} \cdot \vec{B}(\vec{r}, t)
$$

is the Hamiltonian and $\vec{\sigma}$ is the $3 \mathrm{D}$ vector of Pauli matrices. In $1 \mathrm{D}$ its solution can be written in terms of the 2D "spinor" $|\chi(x, t)\rangle$ as

$$
\psi(x, t)=e^{i k x-i \omega t}|\chi(x, t)\rangle
$$

where the exponential corresponds to a neutron moving in the $x$ direction with momentum $\hbar k=m v$ and kinetic energy $E=\hbar \omega=\hbar^{2} k^{2} /(2 m)=\frac{1}{2} m v^{2}$. As follows, by substitution of $\psi(x, t)$ - as written above - into the Schrödinger equation with (2) for the Hamiltonian, $|\chi(x, t)\rangle$ satisfies the so-called Larmor equation for spinors:

$$
\frac{d}{d t}|\chi(x, t)\rangle=\frac{1}{2} i \vec{\sigma} \cdot \gamma \vec{B}(x, t)|\chi(x, t)\rangle .
$$

We prefer to discuss the solution in terms of the corresponding macroscopic 3D spin expectation $\vec{S}(x, t)=$ $\langle\chi(x, t)|\vec{\sigma}| \chi(x, t)\rangle$. In the limit that the neutron's Zeeman energy $\mu_{n} B$ is much less than its kinetic energy $\hbar^{2} k^{2} /(2 m)$, which for thermal neutrons in fields as in our flippers is amply satisfied, Eq. (3) can be written in terms of $\vec{S}(x, t)$ as the "Larmor equation":

$$
\frac{d}{d t} \vec{S}(x, t)=\gamma \vec{S}(x, t) \times \vec{B}(x, t) .
$$

The solution is given in terms of $(3 \times 3)$ rotation matrices denoted $\mathcal{R}_{\vec{m}}(\alpha)$, where $\vec{m}$ is the unit vector along the rotation axis and $\alpha$ the rotation angle. In general $\vec{m}$ is $\hat{x}, \hat{y}$, or $\hat{z}$. To perform the calculations involved, one uses the geometric relation:

$\mathcal{R}_{y}^{-1}(\pi) \mathcal{R}_{z}(\varphi)=\mathcal{R}_{z}^{-1}(\varphi) \mathcal{R}_{y}^{-1}(\pi) \quad$ (+ cycl. variants $)$

which indicates that you can "push" $\mathcal{R}_{y}^{-1}(\pi)$ through $\mathcal{R}_{z}(\varphi)$, provided you invert $\mathcal{R}_{z}$ to $\mathcal{R}_{z}^{-1}$.

\section{A. Solving the Larmor equation for an adiabatic RF flipper}

An adiabatic RF flipper consists of a homogeneous DC magnetic field of strength $B_{0}$, with superimposed a gradient $B_{g r}(x)$, along the $z$ axis. Inside this field sits an RF coil of length $\ell$ generating an oscillating field $B_{R F}(x, t)$ along the $x$ axis at the resonance frequency $\omega=\gamma B_{0}$. Figure 1 gives these field contributions as a function of $x$, as they actually are (see 

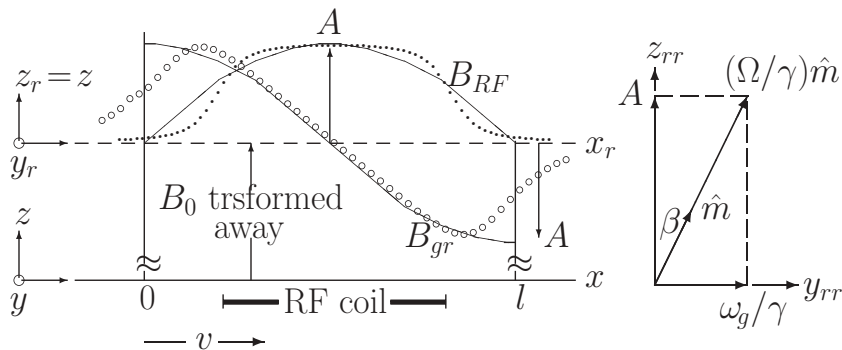

FIG. 2. The fields $B_{g r}(\| z)$ and $B_{R F}(\| x)$ as functions of $x$ : as in the laboratory system (where $B_{R F}$ is twice as strong as shown) and in the system $\left(x_{r}, y_{r}, z\right)$ rotating at frequency $\omega=\gamma B_{0}$ about $z$ : actual $(\mathrm{ooo} / \cdots)$ and idealized (lines). Over length $l$ the effective field (with amplitude $A$ ) for one neutron rotates from $z$ to $-z$ about an axis in the $x y$ plane. (Right) Geometric relation between the "fields" $\omega_{g} / \gamma$, $(\Omega / \gamma) \hat{m}$, and $A$ and the angle $\beta$ occurring in (9).

Sec. IV), and idealized: zero for all $x$, except in the interval $0<x<l$, where they are described as:

$$
\begin{gathered}
B_{0}(x)=B_{0} \hat{z}(\text { hom. field }) \\
B_{R F}(x, t)=2 A \sin (\pi x / l) \cos (\omega t+\varphi) \hat{x}(\text { RF field }) \\
B_{g r}(x)=A \cos (\pi x / l) \hat{z} \text { (grad. field). }
\end{gathered}
$$

The amplitude $2 A$ of the RF field has on purpose been chosen twice the amplitude of the gradient field. The length $l$ corresponds roughly to the length $\ell$ of the RF coil.

A neutron with velocity $v$ along the $x$ axis and spin $\vec{S}_{0}$ enters the idealized configuration of Fig. 2 at time $t=t_{0}$. The solution $\vec{S}(t)$ of the Larmor equation (4) reads (see Appendix):

$$
\begin{aligned}
\vec{S}(t)= & \mathcal{R}_{z}^{-1}(\omega t+\varphi) \mathcal{R}_{y_{r}}\left(\omega_{g} x / v\right) \mathcal{R}_{x_{r}}^{-1}(\beta) \mathcal{R}_{z}^{-1}(\Omega t) \\
& \times \mathcal{R}_{x_{r}}(\beta) \mathcal{R}_{z}(\varphi) \vec{S}_{0},
\end{aligned}
$$

where $\varphi$ is the phase of the RF field at $t_{0}$ and $\omega_{g}=\pi v / l$ the geometric frequency. The diagram in Fig. 2 expresses the relation between the quantities $\omega_{g}, \beta$, and $\Omega$ in (9) and the amplitude $A$. The matrices $\mathcal{R}_{x_{r}}, \mathcal{R}_{x_{r}}^{-1}$, and $\mathcal{R}_{y_{r}}$ represent rotations around the $x_{r}$ and $y_{r}$ axes (at $t_{0}$ at $\varphi$ toward the laboratory system) of the "rotating system" $\left(x_{r}, y_{r}, z_{r}=z\right)$ which rotates at frequency $\omega=\gamma B_{0}$ around $z$. We point out that, seen in the laboratory system, each neutron encounters a different field configuration, expressed by different matrices $\mathcal{R}_{x_{r}}, \mathcal{R}_{x_{r}}^{-1}$, and $\mathcal{R}_{y_{r}}$.

\section{B. Precession phase after one adiabatic RF flipper}

To find the precession phase of the spin $\vec{S}_{f}$ at the end of the flipper, we substitute $t=l / v$ in (9). The final spin $\vec{S}_{f}$ is commonly discussed as a function of the "adiabaticity parameter"

$$
k_{a}(A)=\gamma A / \omega_{g} \quad\left(\rightarrow k_{a} \pi=\gamma(l / v) A\right),
$$

that is, the ratio between the precession frequency in a field of strength $A$ and the geometric frequency $\omega_{g}$. With the help of Fig. 2 it is seen that the quantities $\beta$ and $\Omega t=\Omega l / v$ in (9) can be expressed in $k_{a}$ as (writing $\sqrt{1+k_{a}^{2}}=\kappa$ )

$$
\sin \beta=\frac{1}{\kappa}, \quad \cos \beta=\frac{k_{a}}{\kappa}, \quad \Omega \frac{l}{v}=k_{a} \pi \sqrt{1+1 / k_{a}^{2}} .
$$

Making these substitutions and applying relation (5) four times, we get instead of (9):

$$
\vec{S}_{f}=\mathcal{Q}\left(\Phi_{0}, \varphi, k_{a}\right) \mathcal{R}_{y_{r}}(\pi) \vec{S}_{0}
$$

with $\Phi_{0}=\gamma(l / v) B_{0}$ and

$$
\mathcal{Q}\left(\Phi_{0}, \varphi, k_{a}\right)=\mathcal{R}_{z}^{-1}\left(\Phi_{0}+\varphi\right) \mathcal{M}\left(k_{a}\right) \mathcal{R}_{z}^{-1}(\varphi),
$$

where the matrix $\mathcal{M}\left(k_{a}\right)$ is given by

$$
\mathcal{M}\left(k_{a}\right)=\mathcal{R}_{x_{r}}\left(\beta\left(k_{a}\right)\right) \mathcal{R}_{z}\left(k_{a} \pi \sqrt{1+1 / k_{a}^{2}}\right) \mathcal{R}_{x_{r}}^{-1}\left(\beta\left(k_{a}\right)\right) .
$$

The $y y$ element of the matrix $\mathcal{Q}\left(\Phi_{0}, \varphi, k_{a}\right)$ refers to the NSE maps below. It can be written:

$$
\begin{aligned}
\mathcal{Q}_{y y}= & {\left[1-\mathcal{O}\left(k_{a}^{-2}\right)\right] \cos \left(\Phi_{0}-k_{a} \pi \sqrt{1+1 / k_{a}^{2}}+2 \varphi\right) } \\
& +\mathcal{O}\left(k_{a}^{-2}\right),
\end{aligned}
$$

where the collections of terms $\mathcal{O}\left(k_{a}^{-2}\right)$ approach 0 for $k_{a} \rightarrow \infty$.

In practice the amplitude $A$ is varied rather than $k_{a}$. Expressed in $A$ and substituting (10) for $k_{a}$, the argument of the cosine in (13) is written

$$
\begin{gathered}
\phi_{f}=\Phi_{0}-\Phi(A)+2 \varphi, \text { with } \\
\Phi(A)=\gamma(l / v) A \sqrt{1+\left(\frac{\pi}{\gamma(l / v) A}\right)^{2}} .
\end{gathered}
$$

For $k_{a} \rightarrow \infty$ (adiabatic limit) the angle $\beta$ goes to 0 and the matrix $\mathcal{Q}\left(\Phi_{0}, \varphi, k_{a}\right)$ will consist purely of $\mathcal{R}_{z}$ 's, because $\mathcal{M}\left(k_{a}\right)$ reduces to $\mathcal{R}_{z}(\gamma(l / v) A)$. Then, (11) reduces exactly to:

$$
\begin{gathered}
\vec{S}_{f}=\mathcal{R}_{z}^{-1}\left(\Phi_{0}-\Phi_{\infty}(A)+2 \varphi\right) \mathcal{R}_{y_{r}}(\pi) \vec{S}_{0}, \quad \text { with: } \\
\Phi_{\infty}(A)=\gamma(l / v) A .
\end{gathered}
$$

Since the phase $\varphi$ of the RF field at the arrival time of successive neutrons increases at rate $\omega$, a beam with stationary polarization (all neutrons equal spin $\vec{S}_{0}$ ) transforms into a beam with spins progressing in time at rate $2 \omega$. So the polarization of this beam is not stationary.

\section{Precession phase after NSE arm with two flippers}

Next we consider an NSE arm consisting of two flippers F1 and F2, a distance $L$ apart, with synchronized RF fields with amplitudes $A_{1}$ and $A_{2}$. For a given neutron the spin after F1 is given by Eq. (11). It arrives at $\mathrm{F} 2$ at $t^{*}=t_{0}+L / v$; the phase of the RF field at that time is $\varphi^{*}$. Between the flippers there is no field (ignoring the guide field), so the spin $\vec{S}_{f f}$ after F2 is found by applying (11) [developed to (15) but taking $\Phi(A)$ instead of $\left.\Phi_{\infty}(A)\right]$ twice:

$$
\begin{aligned}
\vec{S}_{f f} \approx & \mathcal{R}_{z}^{-1}\left(\Phi_{0} \pm \Phi\left(A_{2}\right)+2 \varphi^{*}\right) \mathcal{R}_{y_{r}}( \pm \pi) \\
& \times\left[\mathcal{R}_{z}^{-1}\left(\Phi_{0}-\Phi\left(A_{1}\right)+2 \varphi\right) \mathcal{R}_{y_{r}}(\pi)\right] \vec{S}_{0} .
\end{aligned}
$$

We write $\approx$ because we neglect $\mathcal{O}\left(k_{a}^{-2}\right)$. The \pm signs describe whether the gradient in F2 is parallel or opposite to the gradient in F1. In the latter case the diagram in Fig. 2 mirrors about $z_{r r}$, so the geometric frequency for F2 becomes $-\omega_{g}$, hence $\Phi(A)$ in (14) becomes $-\Phi(A)$. Applying relation (5) once 
again (which is valid, since $y_{r}$ is always $\perp z$ ) gives:

$$
\begin{aligned}
\vec{S}_{f f} \approx & \mathcal{R}_{z}^{-1}\left( \pm \Phi\left(A_{2}\right)+2 \varphi^{*}-\Phi\left(A_{1}\right)-2 \varphi\right) \\
& \times\left[\mathcal{R}_{y_{r}}( \pm \pi) \mathcal{R}_{y_{r}}(\pi)\right] \vec{S}_{0} .
\end{aligned}
$$

The phase difference $\varphi^{*}-\varphi$ in the argument of $\mathcal{R}_{z}^{-1}$ equals $\omega L / v$, so it is independent of the arrival time at flipper F1. Hence, for an incoming beam with stationary polarization (all neutrons equal $\vec{S}_{0}$ ) the spin $\vec{S}_{f f}$ no longer depends on time: it is again stationary. Therefore we can identify the spins $\vec{S}_{0}$ and $\vec{S}_{f f}$ with the polarization vector $\vec{P}$ of the beam, so we write $\vec{P}$ instead of $\vec{S}$. Then, realizing that $\mathcal{R}_{y_{r}}( \pm \pi) \mathcal{R}_{y_{r}}(\pi)=\mathcal{I}$, we can rewrite (17):

$$
\begin{gathered}
\vec{P}_{f f} \approx \mathcal{R}_{z}^{-1}\left(\Phi_{Z F}-\Phi\left(A_{1}\right) \pm \Phi\left(A_{2}\right)\right) \vec{P}_{0} \\
\text { with } \quad \Phi_{Z F}=2 \gamma(L / v) B_{0}
\end{gathered}
$$

and $\Phi(A)$ given by (14). $\Phi_{Z F}$ is a precession phase collected "as if" a field $2 B_{0} \hat{z}$ existed all the way from $x=0$ to $x=L$, while in fact there is no field, hence: "zero field precession" [19]. When $A_{1}=A_{2}=A$ the final precession phase $\phi_{f f}$ is

$$
\begin{gathered}
\phi_{f f}=\Phi_{Z F}-\Phi(A)+\Phi(A)=\Phi_{Z F}(\text { gr. parallel }) \\
=\Phi_{Z F}-2 \Phi(A) . \quad \text { (gr. opposite) }
\end{gathered}
$$

(20) implies that when $A_{1}=A_{2}=A$ and gradients parallel, $\phi_{f f}$ will be independent of $A$ for any $\lambda$. Using a limited power supply for the RF fields, a stable NSE setup can be built, although for low amplitude $A$ the polarization will drop by the factor $\left[1-\mathcal{O}\left(k^{-2}\right)\right]$ in Eq. (13).

When the gradients are parallel, but $A_{1} \neq A_{2}$, (20), including (14), becomes:

$$
\begin{gathered}
\phi_{f f}=\Phi_{Z F}-\gamma \frac{l}{v}\left(A_{1}-A_{2}\right)-\Delta \Phi\left(A_{1}, A_{2}\right) ; \\
\Delta \Phi\left(A_{1}, A_{2}\right)=\frac{1}{\gamma(l / v) A_{1}}-\frac{1}{\gamma(l / v) A_{2}},
\end{gathered}
$$

so an extra phase to $\Phi_{Z F}$ appears. Note the different dependence on $v$ of these terms. Figure 9 gives $\Delta \Phi\left(A_{1}, A_{2}\right)$ as functions of $v$ and $\lambda$ for some values of $A_{2}$, with $A_{1}=$ $1.5 \mathrm{mT}$ and $l=80 \mathrm{~mm}$.

\section{Phase RF field second flipper shifted}

When the gradients are parallel and $A_{1}=A_{2}=A$, but the phase of the RF field in $F 2$ is shifted by $\psi$, the phase $\varphi^{*}$ in Eq. (17) increases by $\psi$, so the final phase (20) becomes:

$$
\phi_{f f}(\psi)=\Phi_{Z F}+2 \psi
$$

So we get an extra precession phase $2 \psi$ of the polarization vector, which does not depend on $\lambda$. The phase $2 \psi$ behaves in a way identical with the geometric phase $\phi_{g 1}$ introduced in Sec. II.

\section{Precession phase in $\mathbf{2} \pi$-twisted coil}

A twisted coil of length $l_{t}$ with a DC field of constant strength $B_{t}$, rotating in the $y z$ plane around $\hat{x}$ over $2 \pi$, is described in a frame $(\tilde{x}, \tilde{y}, \tilde{z})$ rotating synchronously with the field vector, as seen by the neutron. So this frame rotates at the geometric frequency $\omega_{t}=2 \pi /\left(l_{t} / v\right)$ and the coil field
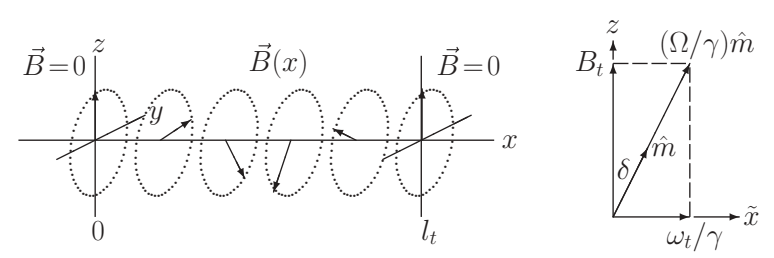

FIG. 3. Field vector in a coil twisted over $2 \pi$ (left) and field diagram in system $\tilde{x}, \tilde{y}, \tilde{z}$ (right).

remains parallel to $\tilde{z}$. Similar to the RF flipper, a pseudofield $\left(\omega_{t} / \gamma\right) \hat{x}$ arises (see diagram in Fig. 3) and the resulting field $(\Omega / \gamma) \hat{m}$ is homogeneous. The solution for the precession is $\overrightarrow{\tilde{P}}(t)=\mathcal{R}_{\vec{m}}^{-1}(\Omega t) \overrightarrow{\tilde{P}}_{0}$ with $\vec{m}=\gamma B_{t} \hat{z}-\omega_{t} \hat{x}$. In the laboratory system this becomes

$$
\vec{P}(t)=\mathcal{R}_{x}^{-1}\left(\omega_{t} t\right) \mathcal{R}_{y}(\delta) \mathcal{R}_{z}^{-1}(\Omega t) \mathcal{R}_{y}^{-1}(\delta) \vec{P}_{0}
$$

We want to discuss this for the full range of the adiabaticity parameter, here defined as:

$$
k_{t} \equiv \gamma B_{t} / \omega_{t}=\gamma B_{t}\left(l_{t} / v\right) / 2 \pi .
$$

Using the diagram in Fig. 3, the quantities $\delta$ and $\Omega t$ in (24) can be expressed in $k_{t}$ as: (writing $\sqrt{k_{t}^{2}+1}=\kappa_{t}$ )

$$
\cos \delta=\frac{k_{t}}{\kappa_{t}}, \sin \delta=\frac{1}{\kappa_{t}}, \Omega t=2 \pi k_{t} \sqrt{1+1 / k_{t}^{2}} .
$$

After substitution, the result at the end of the coil is written: $\vec{P}_{f f}=\mathcal{R}_{x}^{-1}\left(\omega_{t} t\right) \mathbf{P}_{2 \pi}\left(k_{t}\right) \vec{P}_{0}$ with

$$
\mathbf{P}_{2 \pi}\left(k_{t}\right)=\mathcal{R}_{y}\left(\delta\left(k_{t}\right)\right) \mathcal{R}_{z}^{-1}\left(2 \pi k_{t} \sqrt{1+1 / k_{t}^{2}}\right) \mathcal{R}_{y}^{-1}\left(\delta\left(k_{t}\right)\right)
$$

and $k_{t}$ and $\delta$ given by (25) and (26). At the end of the coil the geometric phase $\omega_{t} t=2 \pi$, so $\mathcal{R}_{x}^{-1}\left(\omega_{t} t\right) \rightarrow \mathcal{I}$. The polarization $\vec{P}_{f f}$ of the outcoming beam is stationary. The elements of the matrix $\mathbf{P}_{2 \pi}\left(k_{t}\right)$ as a function of $k_{t}$ are given as the solid lines in Fig. 10.

\section{NSE arm with $2 \pi$-twisted coil between flippers}

To find $\vec{P}_{f f}$ after inserting the coil between the flippers in one NSE arm, we must sandwich (27) in Eq. (16) between the matrices describing F1 and F2. With the twisted coil in the adiabatic limit $\left(k_{t} \rightarrow \infty, \delta \downarrow 0\right)$ this gives (F1 and F2 parallel gradients), applying relation (5) twice:

$$
\vec{P}_{f f}=\mathcal{R}_{z}^{-1}\left(\Phi\left(A_{2}\right)+2 \varphi^{*}-\Omega t-\Phi\left(A_{1}\right)-2 \varphi\right) \vec{P}_{0} .
$$

Assuming $A_{1}=A_{2}=A$, the final precession phase of the polarization vector is

$$
\phi_{f f}=\Phi_{Z F}-\Omega t
$$

with $\Omega t$ given by Eq. (26). So, the twisted coil decreases the precession phase by $\Omega t$, hence the NSE point will shift by this value. In the limit $k_{t} \rightarrow \infty$ this is the dynamic phase when the coil was untwisted.

\section{EXPERIMENTS}

Figure 4 is a sketch of our NSE setup, installed in a thermal beam of the $2 \mathrm{MW}$ HOR Reactor (Delft), made pulsed $(50 \mathrm{~Hz})$ by a chopper [21]. The available spectrum is 


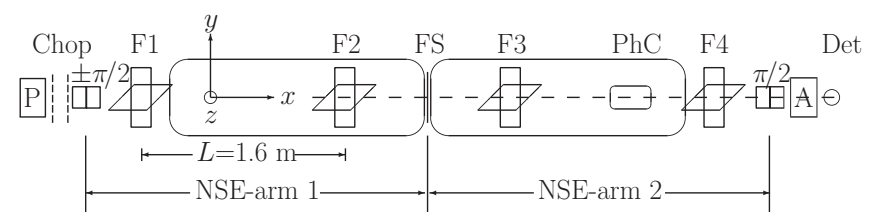

FIG. 4. Top view of our NSE balance with TOF data collection in time-of-flight mode.

$0.1<\lambda<0.8 \mathrm{~nm}$. Data are collected in the time-of-flight mode over $7.6 \mathrm{~m}$ flight path in 500 time channels of $40 \mu \mathrm{s}$ at a rate of 50 frames/s. In data processing the channels are "binned" in groups of five. The ovals are the guide field coils producing a vertical field of $\pm 0.5 \mathrm{mT}$. The device FS is a current screen for a stepwise transition from $+z$ to $-z$ field. $\mathrm{PhC}$ is the phase coil producing a field $\| z$ over $100 \mathrm{~mm}$.

The $\pi / 2$ flippers are each composed of $V$ coils [22]. They are constructed to prepare and analyze the component of the polarization vector along $y$. This means that the $y y$ element of the matrix indicated in Eq. (18) is measured. To measure the degree of polarization, the first flipper is operated as a + and $-\pi / 2$ flipper successively. Figure 5 gives a typical result.

The RF coils in the adiabatic RF flippers F1-F4 $(\ell=66 \mathrm{~mm}$, diameter $=32 \mathrm{~mm}$, wind. density $=5 / \mathrm{cm})$ are operated at $1.1 \mathrm{MHz}\left(B_{0}=36.2 \mathrm{mT}\right)$. Their phases and current amplitudes are monitored on a four-channel oscilloscope. The field profiles in Fig. 2 and the maps of $P(\Delta B, 1 / \lambda)$ in Figs. 6, 7, and 13 are measured with the RF coils carrying a 15-A RF current (giving $A \approx 1.5 \mathrm{mT}$ ) and the gradient field coils carrying a $1.5-\mathrm{A}$ DC current (making a change in $B_{0}$ by $\pm 1.8 \mathrm{mT}$ over the length of the RF coils). For $\lambda=0.1 \mathrm{~nm}$ this "routine setting" gives $k_{a} \approx 2$ averaged over the length of the flipper. For the purpose of NSE-small-angle neutron scattering measurements the poles of the DC magnets are shaped as parallelograms; here this shape is not relevant.

\section{A. NSE with RF fields in arm 2 out of phase}

The NSE group, integrated over wavelength $\left(\lambda_{e f f} \approx\right.$ $0.22 \mathrm{~nm}$ ), was measured at various settings of the phase difference $\psi$ of the RF fields in flippers F3 and F4 in NSE $\operatorname{arm} 2$, while $\mathrm{F} 1$ and F2 in arm 1 were kept in phase. The result
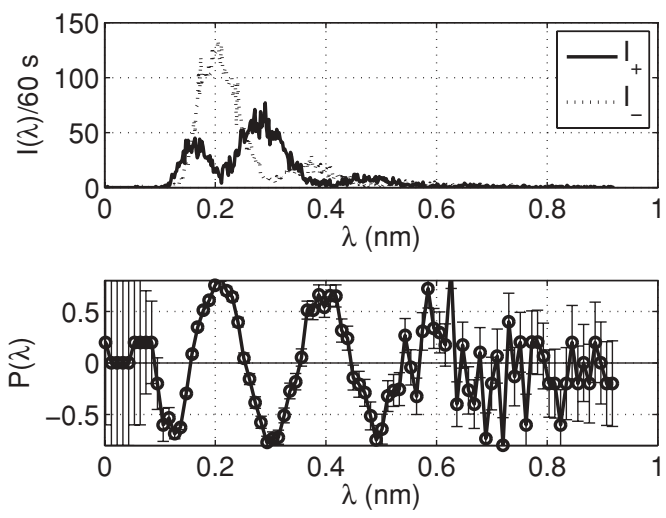

FIG. 5. Intensities and polarization (with statistical errors; binned in groups of five channels) as a function of $\lambda$ in the setting indicated by the line $\mathrm{S}$ in Fig. 6(b).
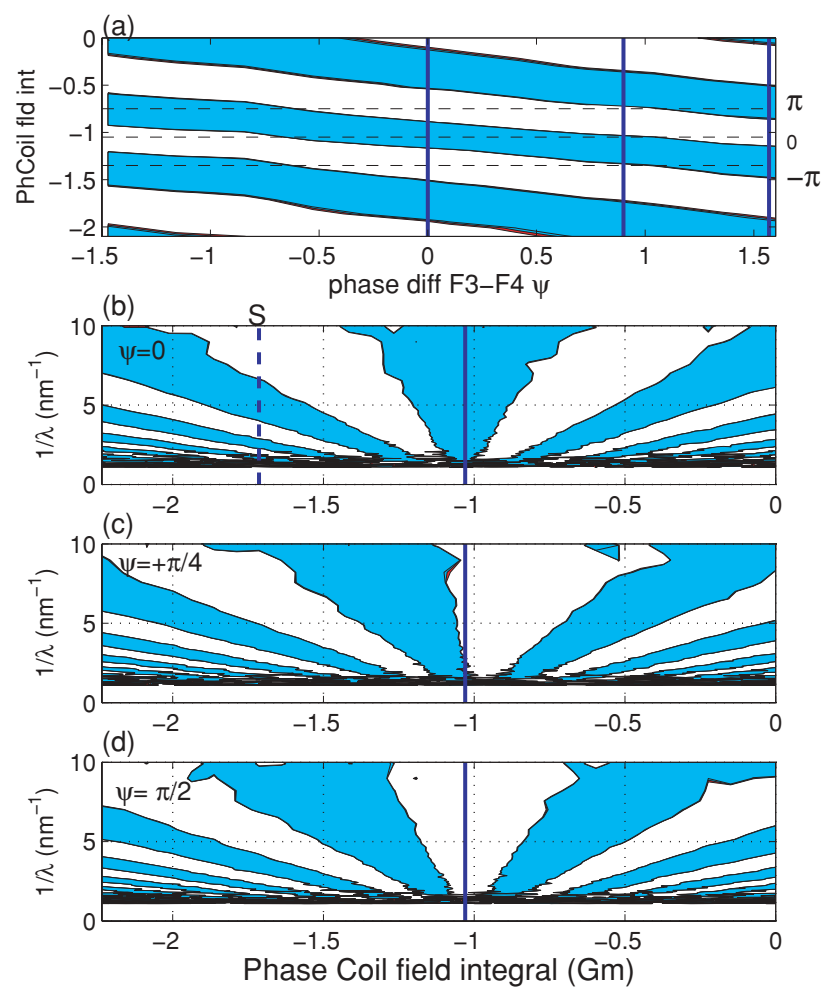

FIG. 6. (Color online) (a) NSE groups, integrated over wavelength, mapped against the phase difference $\psi$ between the RF fields in flipper F3 and F4. (b-d) NSE maps $P(\Delta B, 1 / \lambda)$ for $\psi$ corresponding to the vertical lines in (a). They have the same symmetries as the maps in Fig. 1 for $\phi_{g}=0, \pi / 2$, and $\pi$. The vertical lines mark the common symmetry point. The line $\mathrm{S}$ refers to the data shown in Fig. 5 (dark: $P>0$, white: $P<0$ ).

is given as the map in Fig. 6(a). The dashed lines indicate a precession phase equal to $\pm \pi$ for the effective wavelength $\lambda_{\text {eff }}$. So for $\psi= \pm \pi / 2$ (left and right edge), the NSE group is shifted by $\mp \pi$, as predicted by Eq. (23).

Figures 6(b)-6(d) are maps $P(\Delta B, 1 / \lambda)$ obtained by analyzing the TOF data to $\lambda$. Their analogy with the panels in Fig. 1 shows that the parameter $\psi$ acts as twice the phase $\phi_{g 1}$ in Eq. (1).

\section{B. Reversing one or two gradients in NSE arm 2}

As pointed out in subsection IIIC, reversing the gradient in the first RF flipper in one arm will shift the NSE point by twice the dynamic phase $\Phi(A)$ given in Eq. (14), from the position predicted by (20) to the one predicted by (21). This is confirmed by the positions of the NSE groups in Fig. 7(a) taken integrated over wavelength, with the gradients in NSE arm 1 set parallel $(++)$. The gradients in arm 2 were set in all combinations,,+-++-- , and -+ , as published earlier [17]. Including NSE arm 1, the full configurations are denoted $++++\cdots++--$. For ++++ and ++-the dynamic phase term $\Phi(A)$ cancels [see Eq. (20) with (14)] and NSE is found at phase coil field $\approx 0$. For +++- and ++-+ the NSE point is shifted by $\pm 2 \Phi(A)$ [Eq. (21)], where $\Phi(A)$ [practically equal to $\Phi_{\infty}(A)$ in (15)] is $\approx 10 \times 2 \pi$ (for $\lambda_{e f f}$ ). 

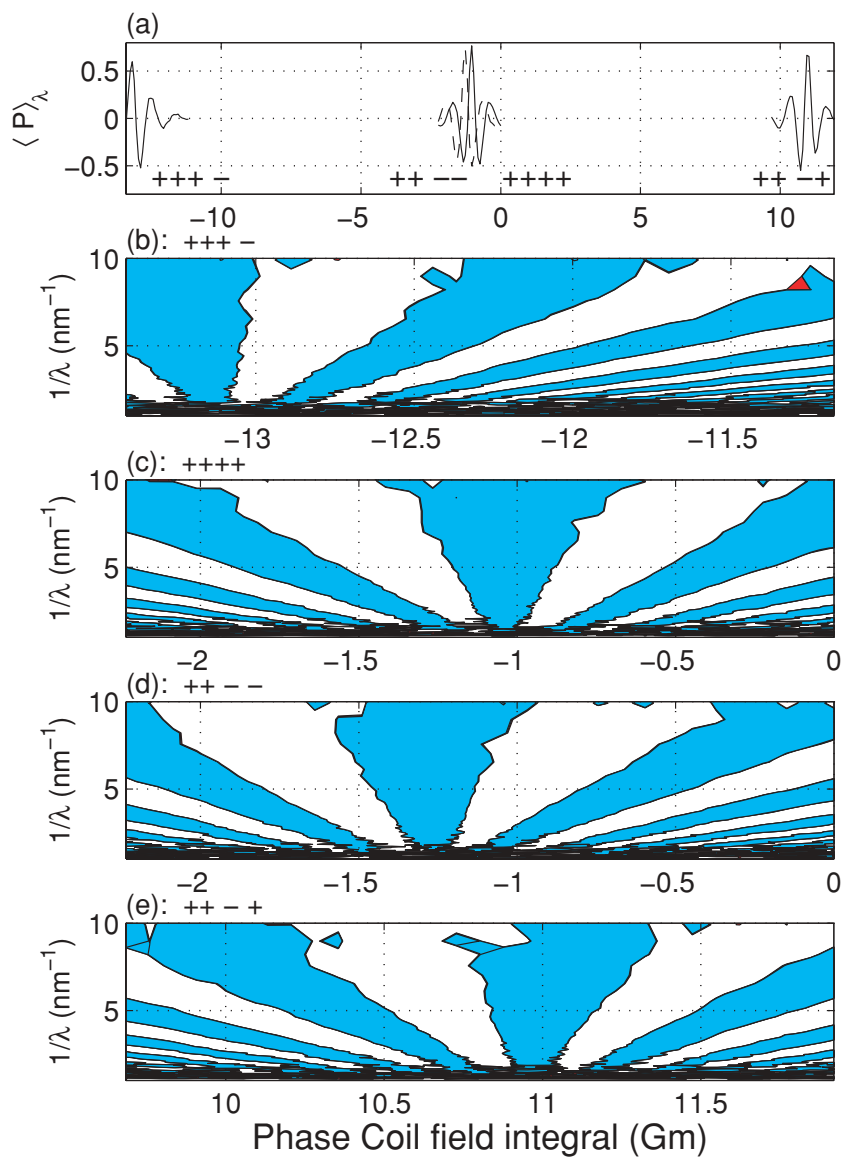

FIG. 7. (Color online) (a): Position of NSE-groups (white beam) for a setup with gradients in F1 and F2 in NSE- arm 1 parallel, combined with all configurations of the gradients in F3 and F4 in NSEarm 2. (b-e) The NSE groups in (a) processed into maps $P(\Delta B, 1 / \lambda)$. Their horizontal scale is extended in comparison with (a) (dark: $P>$ 0; white: $P<0$ ).

On differentiating to $\lambda$, we obtain polarization maps $P(\Delta B, 1 / \lambda)$ similar to Fig. 1 (bottom row). They are shown in Figs. 7(b) $-7(\mathrm{e})$. For ++++ and ++-- the map is symmetric around the NSE point. For +++- and ++-+ it is antisymmetric in opposite ways, which, by analogy with Fig. 1, shows a net geometric phase $\phi_{g}$ at first sight of approximately $\pi / 2$ and $-\pi / 2$, respectively.

The true geometric phases are recognized as follows. NSE arm 1, with gradients $(++)$, has $\pi$ for an argument in the matrix $\mathcal{R}_{y_{r}}$ in (17) for both flippers F1 and F2. So at the end of this arm the field $\vec{B}$ (and the adiabatically following polarization) has described a closed loop in space covering a solid angle 0. By Berry's statement no geometric phase is picked up: $\phi_{g 1}=0$. NSE arm 2, in the configuration $(++)$ or $(--)$, gives the geometric phase $\phi_{g 2}=0$ for the same reason. In the configuration (+-) the first flipper has $\pi$ for an argument in $\mathcal{R}_{y_{r}}$, the second flipper $-\pi$. So the field vector $\vec{B}$ follows a full great circle covering solid angle $2 \pi$. By Berry's argument this gives a geometric phase $\phi_{g 2}=2 \pi(\equiv 0)$. By analogy the configuration (-+) gives the same. So for all gradient configurations of NSE arms 1 and 2 the net geometric phase $\phi_{g}$ is zero: there is no reason why for +++- and ++-+ the map $P(\Delta B, 1 / \lambda)$ should be antisymmetric.
The lower part of Figs. 7(b) and 7(d) gives a hint that this map for these configurations is indeed symmetric: as $1 / \lambda$ decreases ( $\lambda$ increases), $P(\Delta B, 1 / \lambda)$ tends to become symmetric. This means that the term $\Delta \Phi\left(A_{1}, A_{2}\right)$ in Eq. (22) disappears in the measured net phase $\phi_{f f}$. Apparently, for low wavelength the amplitude $A$ of the flippers F3 and F4 in NSE arm 2 is too small to suppress this term. This is discussed further in the next section, where the amplitude in F4 is deliberately reduced.

\section{Varying amplitude $A_{2}$ in second flipper NSE arm 2}

We measured NSE groups, in gradient configuration $(++++)$, at various amplitudes $A_{2}$ of the rotating field (i.e., $B_{g r}$ and $B_{R F}$ ) in flipper F4, with the flippers F1-F3 in the "routine setting" specified in Sec. IV (so they are in the adiabatic regime for most of the neutron spectrum).

Figure $8(a)$ is the map $P(\Delta B, 1 / \lambda)$ measured with flipper F4 also in the "routine setting." [This map should be symmetric around the NSE point. This experiment was done in a separate experimental run with new (inaccurate) setting of the parameters. Apparently, some asymmetry by a net geometric phase was present.]

Figures $8(\mathrm{~b})$ and $8(\mathrm{c})$ give $P(\Delta B, 1 / \lambda)$ measured at lower values of the amplitude $A_{2}$ in F4. Here Fig. 7(a) is projected, after correction for the shift of the NSE point due to the term $\gamma(l / v)\left(A_{1}-A_{2}\right)$ in Eq. (22) arising from the reduction of $A_{2}$. The influence of the term $\Delta \Phi\left(A_{1}, A_{2}\right)$ (i.e., the reduction of
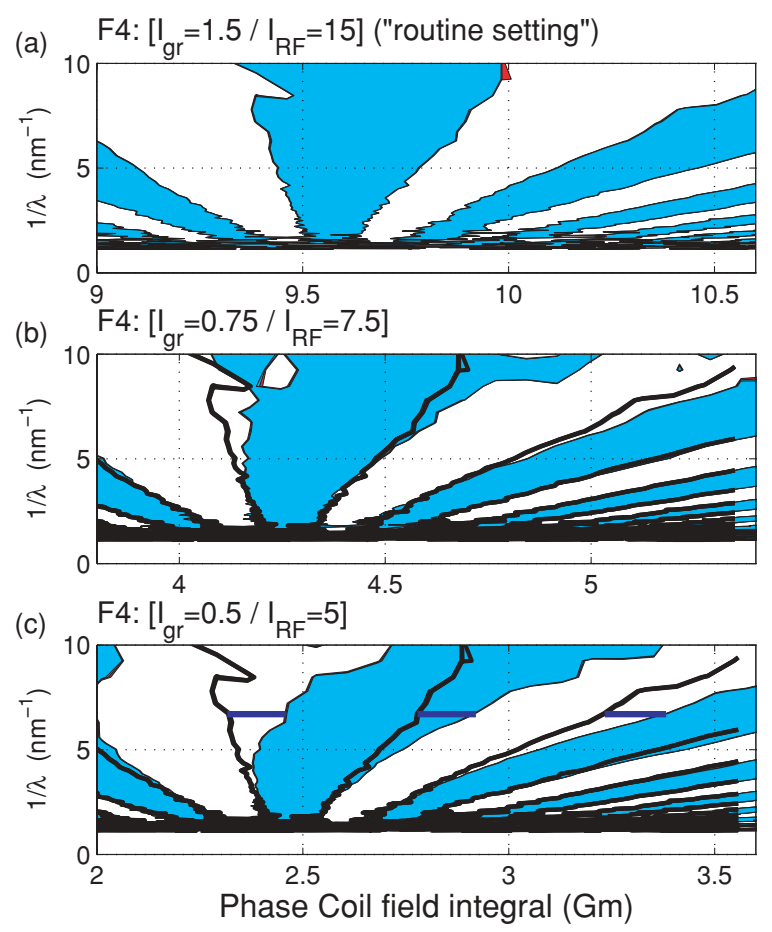

FIG. 8. (Color online) Varying amplitude $A_{2}$ of the rotating field in flipper F4 with F1-F3 kept in "routine setting" $\left[I_{g r}=1.5 A / I_{R F}=\right.$ $15 \mathrm{~A}]$, gradient configuration ++++ . (a) Map $P(\Delta B, 1 / \lambda)$ with F4 also in "routine setting." (b-c) $I_{g r}$ and $I_{R F}$ in F4 reduced by 0.5 and 0.33 (dark: $P>0$; white: $P<0$ ). The horizontal bars mark the phase difference. 

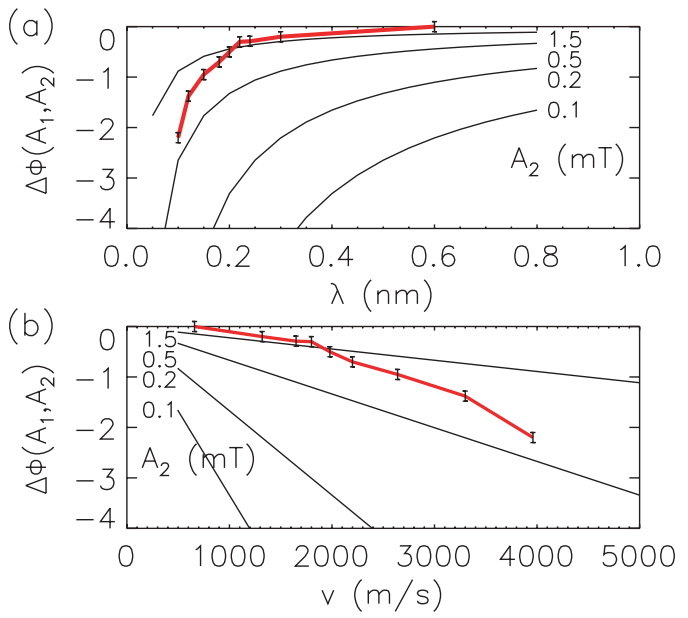

FIG. 9. (Color online) Approach of the term $\Delta \Phi\left(A_{1}, A_{2}\right)$ [Eq. (22)] to 0 as $\lambda$ increases (a) or velocity $v$ decreases (b). Red lines with error bars: read from Fig. 8(c). Black lines: calculated with Eq. (22) for the indicated values of the amplitude $A_{2}$ in F4, with amplitude $A_{1}$ in $\mathrm{F} 3$ at $1.5 \mathrm{mT}$ and length rotating field $l=80 \mathrm{~mm}$.

the net geometric phase in NSE arm 2 to 0 ) can be followed from top $(\lambda=0.1 \mathrm{~nm})$ to bottom $(\lambda=1 \mathrm{~nm})$ : in the bottom (high $\lambda$, so high $k_{a}$ ) the patterns come in phase.

In Fig. 9 the phase difference between the settings for F4 in Figs. 8(a) and 8(c) is plotted as a function of $\lambda$ and $v$. For comparison with theory we add as full lines the outcome of $\Delta \Phi\left(A_{1}, A_{2}\right)$ in Eq. (22) for amplitude $A_{1}$ in F3 equal to $1.5 \mathrm{mT}$ and amplitude $A_{2}$ in $\mathrm{F} 4$ equal to the values given in the figure. The length of the flippers is taken as $l=80 \mathrm{~mm}$.

The figure demonstrates that in an NSE experiment we can follow the development of the geometric phase produced by one flipper (F4) on increasing the amplitude $A$ of its rotating field. It does, however, not follow the prediction of Eq. (22). This is not surprising, since this equation is based on the idealized shape of Fig. 2 for the RF and gradient field.

\section{D. $2 \pi$-twisted coil in NSE arm 1}

As an alternative way to introduce a geometric phase, we mounted two coils of circular cross section in NSE arm 1, each twisted over $\pi$ (Fig. 4) to make one $2 \pi$ coil, similar to those in Refs. [4] and [6]. They were inserted inside a mumetal tube for flux short-circuiting. The coils had a diameter of $60 \mathrm{~mm}$ and wind. density of $1 \mathrm{~mm}^{-1}$; their combined length was $l_{t}=300 \mathrm{~mm}$.

Before the NSE experiment, the matrix $\mathbf{P}_{2 \pi}$ given in Eq. (27) was checked, varying the current $I_{t}$. This was done in the instrument PANDA installed at the HOR in Delft for 3D polarization analysis with a monochromatic beam $(\lambda=$ $0.20 \mathrm{~nm}$ ) in a surrounding field zero.

Figure 10 shows the result, plotted as a function of current $I_{t}$. To compare with $\mathbf{P}_{2 \pi}\left(k_{t}\right)$ according to Eq. (27), the current $I_{t}$ is converted using (25) into $k_{t}$, from the field $B_{t}$ based on the current $I_{t}$ and the winding density. After reducing the calculated field by 0.9 (because the actual field is some less) the agreement between Eq. (27) and the experimental results is good.
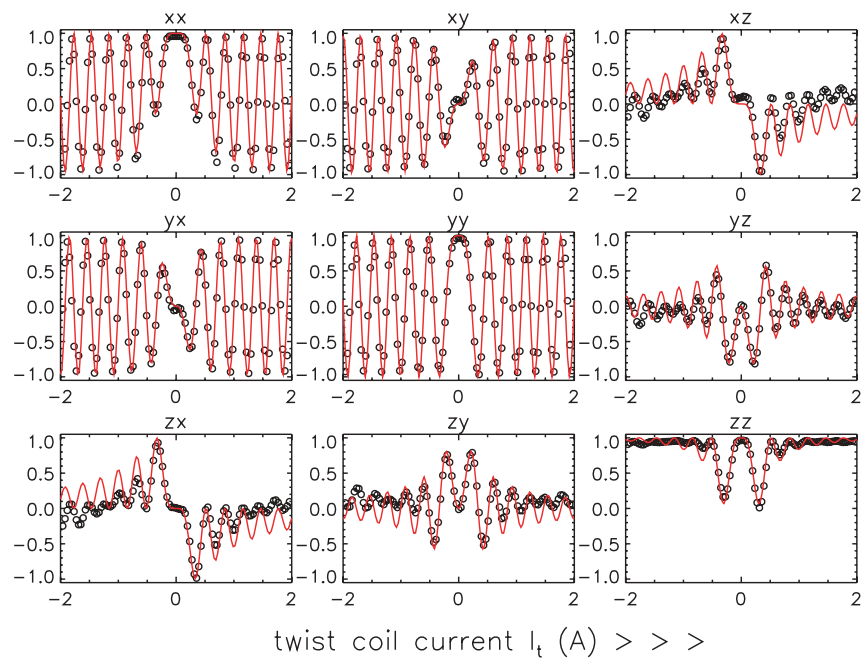

FIG. 10. (Color online) The matrix $\mathbf{P}_{2 \pi}$ according to Eq. (27) (lines) and measured on PANDA (ooo) with a monochromatic beam $(\lambda=0.20 \mathrm{~nm})$.

The precession phase is "measured" from the elements of $\mathbf{P}_{2 \pi}$ by taking

$$
\phi=\tan ^{-1}\left(\left[\mathbf{P}_{2 \pi}\right]_{x y} /\left[\mathbf{P}_{2 \pi}\right]_{y y}\right) .
$$

(This is the only combination from the elements $(x x, x y, y x, y y)$ giving a result without need for corrections for different quadrants). It is shown in Fig. 11 as ++ . The dotted line $\left(\phi=2 k_{t} \pi\right)$ is the dynamic precession phase, if the coil were untwisted. When it is subtracted, we get the full lines/circles. The geometric phase is seen to develop to $\pm 2 \pi$ as $\left|k_{t}\right|$ increases. For a coil of reversed twist (which was not available) this line would mirror about the horizontal axis.

Next, the coil was mounted in arm 1 of the NSE setup in gradient configuration ++++ . The NSE group $P(B)$ integrated over wavelength was measured at various currents $I_{t}$. Equation (28), with (26), implies that the NSE group $P(B)_{I_{t} \neq 0}$ (measured for $I_{t} \neq 0$ ) will be shifted from the group

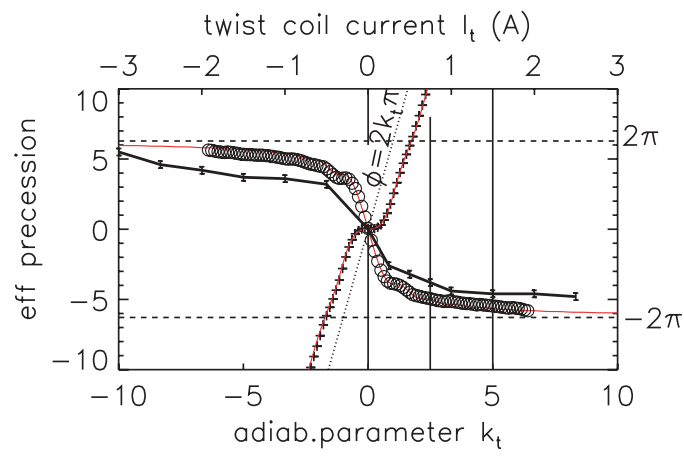

FIG. 11. (Color online) Precession phase in the $2 \pi$-twisted coil as a function of current $I_{t}$. Coil in PANDA: (29) applied to the data in Fig. $10(++)$; after subtracting dynamic phase (oo); (29) applied to the elements of $\mathbf{P}_{2 \pi}$; Eq. (27), (red) lines. Coil in NSE arm 1: phase difference between the patterns $P(B)_{I_{t} \neq 0}$ and $P(B)_{I_{t}=0}$ at various $I_{t}$ (thick lines with bars). This phase difference is the geometric phase. The vertical lines indicate the values of $I_{t}$ of the patterns $I(B)$ in Fig. 12. 

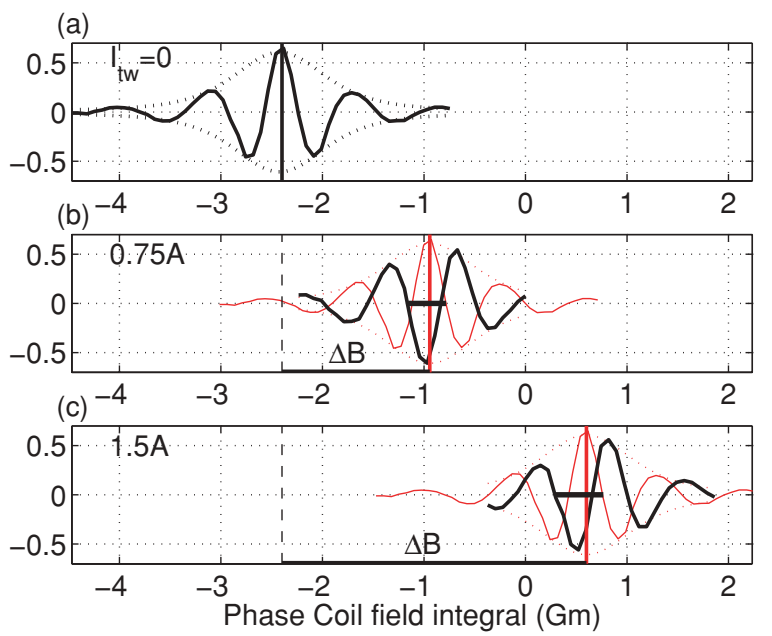

FIG. 12. (Color online) Spin-echo groups $P(B)$ with a $2 \pi$-twisted coil in the NSE arm 1 for currents $I_{t}=0,0.75$, and $1.5 \mathrm{~A}$, integrated over wavelength. The full lines in all panels are the measurements; the thin (red) lines in (b) and (c) are the result in (a) shifted by the dynamic phase $2 \pi k_{t}$. The phase difference (thick bars) between these lines is the geometric phase.

$P(B)_{I_{t}=0}$ (for $I_{t}=0$ ) by a value $\Delta B$ given by $c_{P h} \Delta B=\Omega t=$ $2 \pi k_{t} \sqrt{1+1 / k_{t}^{2}}$.

For each current $I_{t}$ we subtracted the dynamic phase $2 \pi k_{t}$ by shifting the group $P(B)_{I_{t}=0}$ by a value $\Delta B=\alpha I_{t}$, proportional to $I_{t}$. The empirical constant $\alpha$ was fitted such
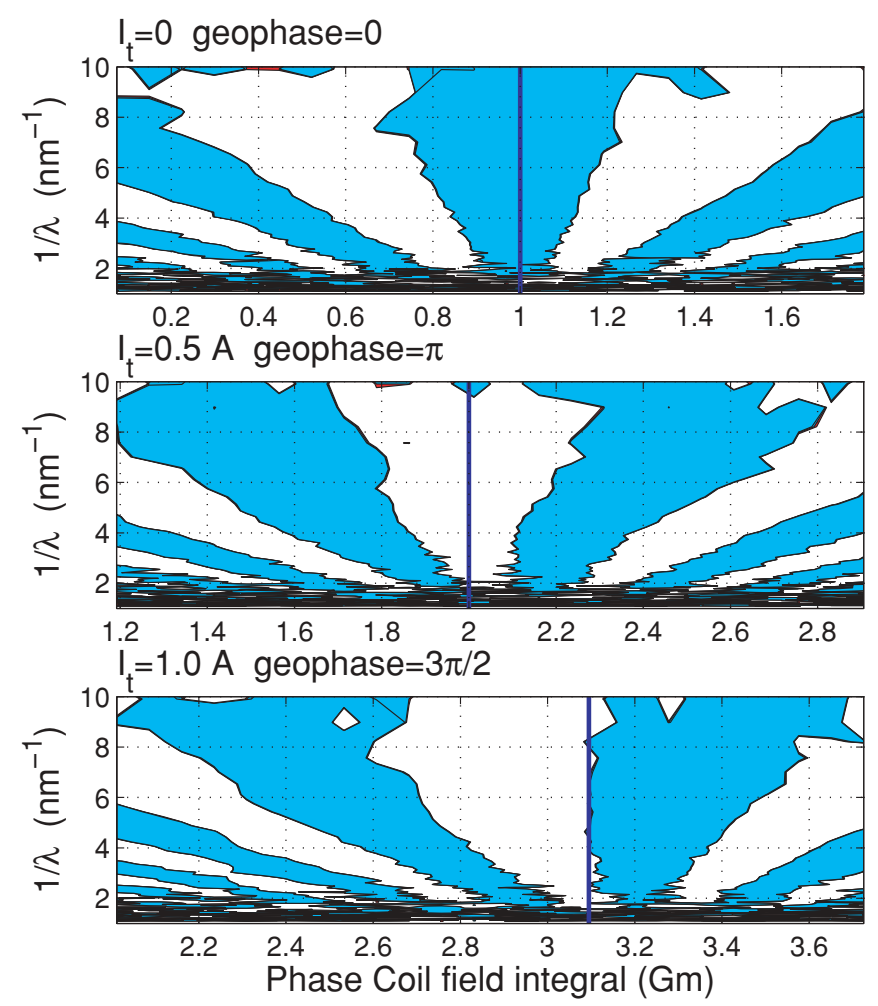

FIG. 13. (Color online) Maps of $P(\Delta B, \lambda)$, config. ++++ , with $2 \pi$-twisted coil in NSE arm 1 , for currents $I_{t}$ such that the geometric phase $=0, \pi$, and $\frac{3}{2} \pi$ (vertical lines in Fig. 11). Symmetries agree with Fig. 1 for $\phi_{g}=0, \pi$ (and $\frac{3}{2} \pi$ ). that for all currents $I_{t}$ the envelop ( $\cdots$ line) of the shifted group $P(B)_{I_{t}=0}$ becomes an envelope for the measured NSE group $P(B)_{I_{t} \neq 0}$. This is illustrated in Fig. 12 for $I_{t}=0.75$ and $1.5 \mathrm{~A}$.

It appears that the NSE patterns $P(B)_{I_{I} \neq 0}$ inside the envelope are shifted with respect to the shifted patterns $P(B)_{I_{t}=0}$. This shift corresponds to the geometric phase picked up in the twisted coil. The thick bars in Figs. 12(b) and 12(c) show that for $I_{t}=0.75 \mathrm{~A}$ and $1.5 \mathrm{~A}$ this shift (for $\lambda_{e f f}$ ) is about 0.5 and 0.7 of a period $2 \pi$, respectively.

The phase differences thus found are added in Fig. 11 as the thick line with error bars. It shows how the geometric phase develops on increasing the current in the $2 \pi$-twisted coil, when it is inserted in NSE arm 1. At either end of Fig. 11 the geometric phase fails to develop to full $2 \pi$. This is because in the NSE experiment the coil is embedded in the guide field of $0.5 \mathrm{mT}$ penetrating into its ends, thereby reducing its overall twist by $10-20 \%$.

In Fig. 13 the NSE patterns corresponding to $\phi_{g}=0, \pi$, and $\simeq 3 \pi / 2$ (that is for $I_{t}=0,0.5$, and $1.0 \mathrm{~A}$ ) are given as maps $P(\Delta B, \lambda)$, analogous to Fig. 1. Their (anti)symmetries agree with the patterns in Fig. 1 based on Eq. (1) for these values for the geometric phase $\phi_{g}$. The conclusion is that the geometric phase picked up in a DC device agrees with the one picked up in a pair of adiabatic/RF flippers.

\section{SUMMARY AND CONCLUSION}

We investigated the NSE pattern for a NSE setup for a polychromatic neutron beam, with each NSE arm made up of 2 adiabatic/gradient $R F$ flippers some distance apart.

In the situation where no net geometric phase remains in NSE, we found a symmetric NSE pattern, as expected. To verify the expected distorted pattern when a net geometric phase remains, we introduced a phase difference $\psi$ between the RF fields in the NSE arm 2. The observed asymmetry in the NSE pattern as a function of $\psi$ confirms the calculation. This finding has the practical benefit that one can exploit the parameter $\psi$ to correct an inadvertent asymmetry in a NSE setup, which might arise, e.g., by the $\pi / 2$ flippers in the beginning and the end.

The suspicion of a geometric phase introduced by the flippers themselves was checked by running through all combinations $(++,--,+-$, and -+$)$ of the signs of the gradients in the flippers of arm 2, while keeping the NSE arm 1 in the (++) configuration. Indeed, the symmetry of the NSE pattern is modified; the observed asymmetries correspond to the geometric phase built up in the various configurations. The accompanying (different) positions of the NSE point can be accounted for. It is remarkable that the expected geometric phase is not fully reached, whereas the flippers operate practically in the "adiabatic limit."

We followed the development of a net geometric phase by measuring the NSE patterns with three flippers at amplitudes of gradient [and RF field such that the adiabatic condition holds practically for the full neutron spectrum]. Flipper 4 was operated in the same setting and in various settings with lower amplitudes. Besides the shift of the NSE point due to the 
dynamic phase, the NSE patterns show the development of the geometric phase.

The buildup of a geometric phase was studied with a $2 \pi$-twisted coil in NSE arm 1 and measuring the NSE pattern at various currents in this coil. The position of the NSE pattern, taking the dynamic phase in this coil into account, can be reconstructed. From the difference between the actual and the reconstructed pattern, the geometric phase can be found. The buildup of the geometric phase from 0 to $5 \mathrm{rad}$ for increasing current in this coil can be followed and the asymmetries in the NSE patterns can be accounted for. It confirms the geometric phase found in a stand-alone test of this coil with a monochromatic neutron beam.

\section{ACKNOWLEDGMENT}

One of the authors (S.V.G.) acknowledges support through a grant from TU-Delft. The work is supported in part by the RFBR (project no. 09-02-01023).

\section{APPENDIX: PRECESSION PHASE AFTER 1 ADIABATIC RF FLIPPER}

A neutron with velocity $v$ along the $x$ axis enters the idealized configuration of Fig. 2 with the field given by Eqs. (4)-(6) at time $t=t_{0}$; the phase of the RF field at $t_{0}$ is $\varphi$. Assuming in Eqs. (4)-(6) that $\hat{B}_{g r}=A$ and $\hat{B}_{R F}=2 A$, the time-dependent field $\vec{B}(t) \equiv \vec{B}(x=v t, t)$ experienced by the neutron can be written as:

$$
\begin{aligned}
\vec{B}(x, t)= & B_{0} \hat{z}+A \mathcal{R}_{y}\left(\omega_{g} x / v\right) \hat{z} \\
& +2 A \mathcal{R}_{z}(\omega t+\varphi) \sin \left(\omega_{g} x / v\right) \hat{x},
\end{aligned}
$$

where $\omega_{g}=\pi v / l$ the geometric frequency and $\hat{x}$ and $\hat{z}$ are unit vectors along the axes $x$ and $z$. Because for each neutron the phase $\varphi$ of the RF field at the entrance time differs, this field applies to one specific neutron. The Larmor equation describing the precession reads:

$$
\frac{d}{d t} \vec{S}(t)=\gamma \vec{S}(t) \times \vec{B}(t) .
$$

We go to a frame $\left(x_{r}, y_{r}, z_{r}=z\right)$ rotating at frequency $\omega=\gamma B_{0}$ around the laboratory $\hat{z}$ axis. In this frame the Larmor equation has unchanged shape, but the spin at time $t$ becomes $\vec{S}_{r}(t) \equiv \mathcal{R}_{z}(\omega t+\varphi) \vec{S}(t)$. So this transformation creates a "field" $\omega / \gamma$ along the rotation direction, thus "transforming away" the homogeneous field $B_{0} \hat{z}$. The RF field [the last term in (A1)] is composed of two counter-rotating fields $B_{r, 1}$ and $B_{r, 2}$ of amplitude $A$ each. $B_{r, 1}$ is stationary in the rotating frame; $B_{r, 2}$ rotates back at $2 \omega$. The field (A1) transforms into

$\vec{B}_{r}(x, t)=A\left[\mathcal{R}_{y_{r}}\left(\omega_{g} x / v\right) \hat{z}_{r}+\mathcal{R}_{z}(2 \omega t+2 \varphi) \sin \left(\omega_{g} x / v\right) \hat{x}_{r}\right]$.

The first term is the sum of the gradient field [written as the second term in (A1)] and the stationary field $B_{r, 1}$. The second term is the field $B_{r 2}$, rotating at $-2 \omega$. Because of its (high) frequency $2 \omega$ it is ineffective and hence neglected.

We go to a doubly rotating frame $\left(x_{r r}, y_{r r}=y_{r}, z_{r r}\right)$ which rotates at frequency $\omega_{g}$ around the $y_{r}$ axis of the frame $\left(x_{r}, y_{r}, z_{r}\right)$. In this system the spin becomes $\vec{S}_{r r}(t) \equiv$ $\mathcal{R}_{y_{r}}^{-1}\left(\omega_{g} x / v\right) \vec{S}_{r}(t)$. The field $\vec{B}_{r r}$ is obtained from $\vec{B}_{r}$ by applying $\mathcal{R}_{y_{r}}^{-1}\left(\omega_{g} x / v\right)$ to the remaining first term of (A3). It reduces to $A \hat{z}_{r r}$. By the rotation of the system $\left(x_{r r}, y_{r r}, z_{r r}\right)$ a new "field" component $\omega_{g} / \gamma \hat{y}_{r r}$ arises along $y_{r r}$. Contrary to the first transformation step, this new component does not compensate an existing field. So the field (A3) is transformed into

$$
\vec{B}_{r r}(t)=(\Omega / \gamma) \vec{m}
$$

This is a constant field along the unit vector

$$
\hat{m}=(\gamma A / \Omega) \hat{z}_{r r}+\left(\omega_{g} / \Omega\right) \hat{y}_{r r}
$$

(see field diagram in Fig. 2 in main text) with strength $\Omega / \gamma=$ $\sqrt{A^{2}+\left(\omega_{g} / \gamma\right)^{2}}$. This is of order $A$ (or larger, depending on $\left.\omega_{g}\right)$. So, in the system $\left(x_{r r}, y_{r r}, z_{r r}\right)$ the field $\vec{B}_{r r}$ is reduced to a homogeneous field and the solution for the spin can readily be written:

$$
\vec{S}_{r r}(t)=\mathcal{R}_{\vec{m}}^{-1}(\Omega t) \vec{S}_{r r}\left(t_{0}\right) .
$$

To transform back to the laboratory system we remember that at any time $\vec{S}_{r r}(t)$ is related with $\vec{S}(t)$ according to:

$$
\vec{S}_{r r}(t)=\mathcal{R}_{y_{r}}^{-1}\left(\omega_{g} x / v\right) \mathcal{R}_{z}(\omega t+\varphi) \vec{S}(t) ;
$$

so for $\left(x=0, t=t_{0}\right)$ we have $\vec{S}_{r r}\left(t_{0}\right)=\mathcal{R}_{z}(\varphi) \vec{S}\left(t_{0}\right)$. Solution (A5), back in the laboratory system, then becomes:

$$
\vec{S}(t)=\mathcal{R}_{z}^{-1}(\omega t+\varphi) \mathcal{R}_{y_{r}}\left(\omega_{g} x / v\right) \mathcal{R}_{\vec{m}}^{-1}(\Omega t) \mathcal{R}_{z}(\varphi) \vec{S}\left(t_{0}\right)
$$

To write Eq. (A7) in terms of standard rotation matrices, we introduce the angle $\beta$ (see diagram Fig. 2 in the main text) by $\sin \beta=\omega_{g} / \Omega$. In terms of this angle we write the unit vector $\hat{m}=\mathcal{R}_{x}^{-1}(\beta) \hat{z}$. We can then replace $\mathcal{R}_{\vec{m}}^{-1}(\Omega t)$ with $\mathcal{R}_{x_{r}}^{-1}(\beta) \mathcal{R}_{z}^{-1}(\Omega t) \mathcal{R}_{x_{r}}(\beta)$ and (A7) becomes:

$$
\begin{aligned}
\vec{S}(t)= & \mathcal{R}_{z}^{-1}(\omega t+\varphi) \mathcal{R}_{y_{r}}\left(\omega_{g} x / v\right) \mathcal{R}_{x_{r}}^{-1}(\beta) \mathcal{R}_{z}^{-1}(\Omega t) \\
& \times \mathcal{R}_{x_{r}}(\beta) \mathcal{R}_{z}(\varphi) \vec{S}\left(t_{0}\right) .
\end{aligned}
$$

This is Eq. (9) in the main text.
[1] F. Mezei, C. Pappas, and T. Gutberlet (eds.), Neutron Spin Echo Spectroscopy (Springer, Berlin, 2003), p. 7.

[2] J. Plomp, V. O. de Haan, R. M. Dalgliesh, S. Langridge, A. A. van Well, Physica B 397, 76 (2007).

[3] A. N. Bazhenov, V. M. Lobashev, A. N. Pirozhkov, and V. N. Slusar, Nucl. Instrum. Methods A 332, 534 (1993).
[4] T. Bitter and D. Dubbers, Phys. Rev. Lett. 59, 251 (1987).

[5] M. V. Berry, Proc. R. Soc. London A 392, 45 (1984).

[6] V. I. Bodnarchuk, L. S. Davtyan, and D. A. Korneev, USPEKHI PHIZ. NAUK 166, 185 (1996).

[7] H. Weinfurter and G. Badurek, Phys. Rev. Lett. 64, 1318 (1990). 
[8] D. J. Richardson, A. I. Kilvington, K. Green, and S. K. Lamoreaux, Phys. Rev. Lett. 61, 2030 (1988).

[9] A. G. Wagh, Phys. Lett. A 146, 369 (1990).

[10] Y. Hasegawa, S. Menhart, R. Meixner, and G. Badurek, Phys. Lett. A 234, 322 (1997).

[11] A. Shapere and F. Wilczek (eds.), Geometric Phases in Physics (World Scienticic, Singapore, 1989).

[12] A. Tomita and R. Y. Chiao, Phys. Rev. Lett. 57, 937 (1986).

[13] U. Bonse and H. Rauch (eds.), Neutron Interferometry (Oxford University Press, New York, 1979).

[14] S. Sponar, J. Klepp, R. Loidl, S. Filipp, G. Badurek, Y. Hasegawa, and H. Rauch, Phys. Rev. A 78, 061604 (R) (2008).

[15] A. G. Wagh, V. C. Rakhecha, J. Summhammer, G. Badurek, H. Weinfurter, B. E. Allman, H. Kaiser, K. Hamacher,
D. L. Jacobson, and S. A. Werner, Phys. Rev. Lett. 78, 755 (1997).

[16] A. Ioffe and F. Mezei, Physica B 297, 303 (2001).

[17] S. V. Grigoriev, W. H. Kraan, F. M. Mulder, and M. T. Rekveldt, Phys. Rev. A 62, 063601 (2000).

[18] S. Filipp, J. Klepp, Y. Hasegawa, C. Plonka-Spehr, U. Schmidt, P. Geltenbort, and H. Rauch, Phys. Rev. Lett. 102, 030404 (2009).

[19] R. Golub, R. Gähler, and T. Keller, Am. J. Phys. 62, 779 (1994).

[20] A. Abragam, Principles of Nuclear Magnetism (Oxford University Press, New York, 1961).

[21] A. A. van Well and H. Fredrikze, Physica B 357, 204 (2005).

[22] W. H. Kraan, M. T. Rekveldt, and P. T. Por, Nucl. Instrum. Methods A 300, 35 (1991). 